\title{
MINORS IN RANDOM REGULAR GRAPHS
}

\author{
NIKOLAOS FOUNTOULAKIS, DANIELA KÜHN AND DERYK OSTHUS
}

\begin{abstract}
We show that there is a constant $c$ so that for fixed $r \geq 3$ a.a.s. an $r$-regular graph on $n$ vertices contains a complete graph on $c \sqrt{n}$ vertices as a minor. This confirms a conjecture of Markström 17. Since any minor of an $r$-regular graph on $n$ vertices has at most $r n / 2$ edges, our bound is clearly best possible up to the value of the constant $c$. As a corollary, we also obtain the likely order of magnitude of the largest complete minor in a random graph $G_{n, p}$ during the phase transition (i.e. when $p n \rightarrow 1$ ).
\end{abstract}

\section{INTRODUCTION}

We say that a graph $G$ contains a complete graph on $k$ vertices (denoted by $K_{k}$ ) as a minor if we can obtain a copy of $K_{k}$ after a series of contractions of the edges and deletions of vertices or edges of $G$. We write $K_{k} \prec G$ in this case. Equivalently, $G$ has a $K_{k}$ minor if there are $k$ pairwise disjoint non-empty subsets of $V(G)$ (which we call branch sets) such that each of them is connected and any two of them are joined by an edge. The contraction clique number $\operatorname{ccl}(G)$ of $G$ is the largest integer $k$ such that $G$ has a $K_{k}$ minor.

Originally, the study of the order of the largest complete minor in a random graph was motivated by Hadwiger's conjecture which states that $\operatorname{ccl}(G) \geq \chi(G)$ for any graph $G$. Bollobás, Erdös and Catlin [7] showed that the proportion of graphs on $n$ vertices that satisfy Hadwiger's conjecture tends to 1 as $n$ tends to infinity. For this, they determined the likely value of $\operatorname{ccl}\left(G_{n, p}\right)$ for the random graph $G_{n, p}$ with constant edge probability $p$ and compared this with known results on $\chi\left(G_{n, p}\right)$. Krivelevich and Sudakov [13] investigated $\operatorname{ccl}(G)$ for expanding graphs $G$ and derived the order of magnitude of $\operatorname{ccl}\left(G_{n, p}\right)$ from their results when $p$ is a polynomial in $n$. In [9], we extended these results to any $p$ with $p n \geq c$ for some constant $c>1$, which answered a question from [13. In particular, we showed that if $p n=c$ for some fixed $c>1$ then a.a.s.

$$
\operatorname{ccl}\left(G_{n, p}\right)=\Theta(\sqrt{n}) .
$$

The upper bound is immediate, as for such $p$ a.a.s. the random graph $G_{n, p}$ has $\Theta(n)$ edges and no minor of a graph $G$ can contain more edges than $G$ itself. Here we write that an event regarding a graph on $n$ vertices holds a.a.s. if the probability of this event tends to 1 as $n$ tends to infinity.

Markström [17] had earlier conjectured a similar phenomenon as in (1) for the case of random regular graphs. For any $r \geq 3$ and $n \geq 4$ such that $r n$ is even, we denote by $G(n, r)$ a graph chosen uniformly at random from the set of $r$-regular simple graphs on $n$ vertices. Throughout, we consider the case where $r$ is fixed. The number of edges of $G(n, r)$ is $r n / 2$ and so the same argument as above shows that $\operatorname{ccl}(G(n, r)) \leq 2 \sqrt{r n}$. However the lower bound in (11) does not imply that a random $r$-regular graph satisfies $\operatorname{ccl}(G(n, r))=\Omega(\sqrt{n})$ as the asymptotic structure of $G(n, r)$ is quite different from that of $G_{n, r / n}$ (see for example [20] or Chapter 9 in [11]). Markström [17] proved that $G(n, 3)$ a.a.s. contains a complete minor

Date: November 2, 2018 
of order $k$, for any integer $k \geq 3$ and conjectured that $G(n, 3)$ contains a complete minor of order $\Omega(\sqrt{n})$. In this paper, we verify this conjecture for any $r \geq 3$ :

Theorem 1. There exists an absolute constant $c>0$ such that for every fixed $r \geq 3$ a.a.s. $c \sqrt{n} \leq \operatorname{ccl}(G(n, r)) \leq 2 \sqrt{r n}$.

This result can be combined with results of Łuczak [15] to determine the likely order of magnitude of $\operatorname{ccl}\left(G_{n, p}\right)$ during the phase transition, i.e. when $p n \rightarrow 1$ (see Section 2 for details).

Corollary 2. There exists an absolute constant $c>0$ such that whenever $n p=1+\lambda n^{-1 / 3}$, where $\lambda=\lambda(n) \rightarrow \infty$ but $\lambda=o\left(n^{1 / 3}\right)$, then a.a.s. $c \lambda^{3 / 2} \leq \operatorname{ccl}\left(G_{n, p}\right) \leq 4 \lambda^{3 / 2}$.

Łuczak, Pittel and Wierman [16] previously showed that a.a.s. $\operatorname{ccl}\left(G_{n, p}\right)$ is unbounded for $p$ as in Corollary 2. For smaller $p$ (i.e. when $n p \leq 1+\lambda n^{-1 / 3}$ for some constant $\lambda$ ) they showed that $\operatorname{ccl}\left(G_{n, p}\right)$ is bounded in probability, i.e. for every $\delta>0$ there exists $C=C(\delta)$ such that $\mathbb{P}\left(\operatorname{ccl}\left(G_{n, p}\right)>C\right)<\delta$. As described earlier, values of $p$ which are larger than those allowed for in Corollary 2 but bounded away from 1 are covered in [9]. So altogether, all these results determine the likely order of magnitude of $\operatorname{ccl}\left(G_{n, p}\right)$ for any $p$ which is bounded away from 1.

The results in [16] were proved in connection with the following result on the limiting probability $g(\lambda)$ that $G_{n, p}$ is planar in the above range. The authors proved that if $\lambda$ is bounded, then $g(\lambda)$ is bounded away from 0 and 1 . If $\lambda \rightarrow-\infty$, then $g(\lambda) \rightarrow 1$, whereas if $\lambda \rightarrow \infty$, then $g(\lambda) \rightarrow 0$.

The result in (11) and Theorem 1 raise the question of whether one can extend these results to other (not necessarily random) graphs. A natural class to consider are expanding graphs: A graph $G$ on $n$ vertices is an $(\alpha, t)$-expander if any $X \subseteq V(G)$ with $|X| \leq \alpha n / t$ satisfies $|N(X)| \geq t|X|$, where $N(X)$ denotes the external neighbourhood of $X$.

Problem 3. Is there a constant $c>0$ such that for each $r \geq 3$ every $r$-regular $(1 / 3,2)$ expander satisfies $\operatorname{ccl}(G) \geq c \sqrt{n}$ ?

An answer to the problem would indicate whether expansion alone is sufficient when trying to force a complete minor of the largest possible order in a sparse graph, or whether other parameters are also relevant. Krivelevich and Sudakov [13 showed that we do have $\operatorname{ccl}(G) \geq c \sqrt{n / \log n}$ if $r \geq 10$. (They also considered the case when $r$ is not bounded but grows with $n$.) As observed in [13, this bound can also be deduced from a result of Plotkin, Rao and Smith [19] on separators in graphs without a large complete minor. Kleinberg and Rubinfeld [12] also considered the same problem but with a weaker definition of expansion.

One can ask similar questions as above for topological minors. Topological minors in random graphs were investigated in [6, 7, 1]. An analogue of Problem 3 would be to ask for which values of $\alpha, t, r$ an $r$-regular $(\alpha, t)$-expander on $n$ vertices contains a subdivision of a $K_{r+1}$. We expect that this might not be difficult to prove for fixed $r$ but harder if $r$ is no longer very small compared to $n$.

\section{Proof of Corollary 2}

The upper bound in Corollary 2 will follow from basic facts about minors as well as the structure of $G_{n, p}$. Bollobás [5] (see also [3] or [11]) proved that a.a.s. all the components of $G_{n, p}$, except from the largest one, are either trees or unicyclic. Therefore none of them contains a $K_{4}$ minor. Let $L_{1}\left(G_{n, p}\right)$ denote the largest component of $G_{n, p}$. Given a graph $G$, we define its excess as $\operatorname{exc}(G):=e(G)-|G|+1 .(\operatorname{exc}(G)$ is also called the cyclomatic number of $G$.) Observe that if $H$ and $G$ are connected graphs and $H \prec G$ then $\operatorname{exc}(H) \leq \operatorname{exc}(G)$. 
Since $\operatorname{exc}\left(K_{r}\right)=\left(\begin{array}{l}r \\ 2\end{array}\right)-r+1 \geq r^{2} / 16$ for $r \geq 4$ this implies that if $K_{r} \prec L_{1}\left(G_{n, p}\right)$ for some $r \geq 4$ then $r^{2} / 16 \leq \operatorname{exc}\left(L_{1}\left(G_{n, p}\right)\right)$, or equivalently

$$
r \leq 4 \sqrt{\operatorname{exc}\left(L_{1}\left(G_{n, p}\right)\right)}
$$

Luczak 14] gave a tight estimate on $\operatorname{exc}\left(L_{1}\left(G_{n, m}\right)\right)$, where $G_{n, m}$ is a random graph with $n$ vertices and $m$ edges (i.e. $G_{n, m}$ is chosen uniformly at random among all such graphs). He proved that if $m=n / 2+\bar{\lambda} n^{2 / 3}$, where $\bar{\lambda}=\bar{\lambda}(n) \rightarrow \infty$ but $\bar{\lambda}=o\left(n^{1 / 3}\right)$, then a.a.s. $\operatorname{exc}\left(L_{1}\left(G_{n, m}\right)\right)=(1+o(1)) 16 \bar{\lambda}^{3} / 3$. This trivially implies that if $m=n / 2+\bar{\lambda} n^{2 / 3}+O(\sqrt{n})$ then a.a.s. $\operatorname{exc}\left(L_{1}\left(G_{n, m}\right)\right) \leq 8 \bar{\lambda}^{3}$. Together with the fact that $\left(\begin{array}{c}n \\ 2\end{array}\right) p=n / 2+\lambda n^{2 / 3} / 2+O(1)$ and Proposition 1.12 in [11] this implies that a.a.s. $\operatorname{exc}\left(L_{1}\left(G_{n, p}\right)\right) \leq \lambda^{3}$. But if the latter holds and $K_{r} \prec L_{1}\left(G_{n, p}\right)$ for some $r \geq 4$ then (2) gives $r \leq 4 \lambda^{3 / 2}$. Thus a.a.s. $\operatorname{ccl}\left(G_{n, p}\right) \leq$ $4 \lambda^{3 / 2}$.

For the lower bound in Corollary 2 we will use the following result of Euczak which is contained in the proof of Theorem $5^{*}$ in [15].

Theorem 4. Suppose that $m=n / 2+\lambda n^{2 / 3}$, where $\lambda \rightarrow \infty$ and $\lambda=o\left(n^{1 / 3}\right)$. Then there is a procedure which in any given graph $G$ with $n$ vertices and $m$ edges finds a subdivision of $a$ (possibly empty) 3-regular graph $C=C(G)$ such that a.a.s. $\left|C\left(G_{n, m}\right)\right|=(32 / 3+o(1)) \lambda^{3}$ and conditional on $\left|C\left(G_{n, m}\right)\right|=s$ in this range the distribution of $C\left(G_{n, m}\right)$ is the same as $G(s, 3)$.

Loosely speaking, Theorem 4 implies that a.a.s. $L_{1}\left(G_{n, m}\right)$ contains a subdivision of a random 3-regular graph $G(s, 3)$ where $s=(32 / 3+o(1)) \lambda^{3}$. Together with Theorem 1 this implies that a.a.s.

$$
\operatorname{ccl}\left(G_{n, m}\right) \geq \operatorname{ccl}\left(C\left(G_{n, m}\right)\right) \geq c \lambda^{3 / 2} .
$$

Again Proposition 1.12 from [11] now yields the lower bound of Corollary 2 .

\section{Sketch of Proof of Theorem 1}

We will use a result of Janson [10] which implies that it suffices to find a complete minor in the union of a random Hamilton cycle and a random perfect matching. We split the Hamilton cycle into paths $P_{1}$ and $P_{2}$ of equal length. We further split $P_{1}$ into $k$ connected candidate branch sets $B_{i}$, where $k$ is close to $\sqrt{n}$. Each of these candidate branch sets has size roughly $\sqrt{n}$. We now split $P_{2}$ into sets $\mathcal{P}_{i}$ of disjoint paths. The lengths of the paths in $\mathcal{P}_{i}$ is roughly $3^{i}$, whereas the number of paths in $\mathcal{P}_{i}$ is roughly $n / 9^{i}$. For each pair $\left(B, B^{\prime}\right)$ of candidate branch sets we aim to find a path $P$ in some $\mathcal{P}_{i}$ such that both $B$ and $B^{\prime}$ are joined to $P$ by an edge of the random perfect matching. We let $\mathcal{U}_{i-1}$ denote the set of pairs of candidate branch sets for which we were not able to find such a path $P$ in $\bigcup_{j<i} \mathcal{P}_{j}$. We will show inductively that $\left|\mathcal{U}_{i}\right| \leq\left|\mathcal{U}_{i-1}\right| / 27$ (with sufficiently high probability). By continuing this for $\left(\log _{3} n\right) / 6$ stages and discarding a few atypical branch sets, we eventually obtain the desired minor. This strategy is similar to that of [9]. However, the proof that it works is very different: the argument in [9] was based on a greedy matching algorithm whose analysis crucially relied on the independence of certain events. In the current setting, this no longer works. So instead, in each stage we use Hall's theorem to find a large matching in the bipartite auxiliary graph whose vertex classes are $\mathcal{U}_{i-1}$ and $\mathcal{P}_{i}$ and where a pair $\left(B, B^{\prime}\right)$ is

adjacent to a path $P \in \mathcal{P}_{i}$ if $P$ can be used to join $B$ and $B^{\prime}$ as above. (Actually, it turns out that we need to consider suitable subsets $\mathcal{U}_{i-1}^{\prime} \subseteq \mathcal{U}_{i-1}$ and $\mathcal{S} \subseteq \mathcal{P}_{i}$ for the argument to work.) Though the number $\left|\mathcal{P}_{i}\right|$ of paths decreases in each stage, the increasing path length means that the average degree of a pair $\left(B, B^{\prime}\right)$ in this auxiliary graph remains large (but 
bounded) in each stage and so we can indeed expect to find a large matching. On the other hand, one can show that there might be a significant number of pairs from $\mathcal{U}_{i-1}$ which are isolated in the auxiliary graph. So we cannot hope to get away with just a single stage.

\section{Models OF RANDOM $r$-REGUlaR GRAPHS}

The aim of this section is to show that it suffices to find our complete minor in the union of a random Hamilton cycle and a random perfect matching. To do this, let us first describe the configuration model which was introduced by Bender and Canfield [2] and independently by Bollobás [4. For $n \geq 1$ let $V_{n}:=\{1, \ldots, n\}$. Also for those $n$ for which $r n$ is even, we let $P:=V_{n} \times[r]$. A configuration is a perfect matching on $P$. If we project a configuration onto $V_{n}$, then we obtain an $r$-regular multigraph on $V_{n}$. Let $G^{*}(n, r)$ denote the random multigraph that is the projection of a configuration on $P$ which is chosen uniformly at random. It can be shown (see e.g. [11, p. 236]) that if we condition on $G^{*}(n, r)$ being simple (i.e. it does not have loops or multiple edges), then this is distributed uniformly among the $r$-regular graphs on $V_{n}$. In other words, $G^{*}(n, r)$ conditional on being simple has the same distribution as $G(n, r)$. We also let $G^{\prime}(n, r)$ denote a random multigraph whose distribution is that of $G^{*}(n, r)$ conditional on having no loops. We will use the above along with the following (see Corollary 9.7 in [11]):

$$
\lim _{n \rightarrow \infty} \mathbb{P}\left(G^{*}(n, r) \text { is simple }\right)>0 .
$$

(Of course the above limit is taken over those $n$ for which $r n$ is even.) Let $A_{n}$ be a subset of the set of $r$-regular multigraphs on $V_{n}$. Altogether the above facts imply that if $\mathbb{P}\left(G^{\prime}(n, r) \in\right.$ $\left.A_{n}\right) \rightarrow 0$ as $n \rightarrow \infty$ then $\mathbb{P}\left(G(n, r) \in A_{n}\right) \rightarrow 0$. Indeed, suppose that the former holds. Then

$$
\begin{aligned}
& \mathbb{P}\left(G(n, r) \in A_{n}\right)=\mathbb{P}\left(G^{*}(n, r) \in A_{n} \mid G^{*}(n, r) \text { simple }\right)=\frac{\mathbb{P}\left(G^{*}(n, r) \in A_{n}, G^{*}(n, r) \text { simple }\right)}{\mathbb{P}\left(G^{*}(n, r) \text { simple }\right)} \\
& \text { (4) } \quad \leq \frac{\mathbb{P}\left(G^{*}(n, r) \in A_{n}, G^{*}(n, r) \text { has no loops }\right)}{\mathbb{P}\left(G^{*}(n, r) \text { has no loops }\right) \mathbb{P}\left(G^{*}(n, r) \text { simple }\right)}=\frac{\mathbb{P}\left(G^{\prime}(n, r) \in A_{n}\right)}{\mathbb{P}\left(G^{*}(n, r) \text { simple }\right)} \stackrel{3}{\rightarrow} 0 .
\end{aligned}
$$

This allows us to work with $G^{\prime}(n, r)$ instead of $G(n, r)$ itself.

Let us first assume that $r=3$. The reason for working with $G^{\prime}(n, 3)$ is that we may think of it as being the union of a random Hamilton cycle on $V_{n}$ and a random perfect matching on $V_{n}$. This is made precise by the notion of contiguity. If $\left(\mu_{n}\right)$ and $\left(\nu_{n}\right)$ are two sequences of probability measures such that for each $n, \mu_{n}$ and $\nu_{n}$ are measures on the same measurable space $\Omega_{n}$, then we say that they are contiguous if for every sequence of measurable sets $\left(A_{n}\right)$ with $A_{n} \in \Omega_{n}$ we have $\lim _{n \rightarrow \infty} \mu_{n}\left(A_{n}\right)=0$ if and only if $\lim _{n \rightarrow \infty} \nu_{n}\left(A_{n}\right)=0$. Now let $H(n)+G(n, 1)$ denote the random multigraph on $V_{n}$ that is obtained from a Hamilton cycle on $V_{n}$ chosen uniformly at random by adding a random perfect matching on $V_{n}$ chosen independently from the Hamilton cycle. Janson [10] (see also Theorem 9.30 in [11]) proved that $H(n)+G(n, 1)$ is contiguous to $G^{\prime}(n, 3)$.

Theorem 5. The random 3-regular multigraphs $H(n)+G(n, 1)$ and $G^{\prime}(n, 3)$ are contiguous.

So instead of proving Theorem 1 directly, it suffices to prove the following result.

Theorem 6. There exists an absolute constant $c^{\prime}>0$ such that a.a.s. the random multigraph $H(n)+G(n, 1)$ contains a complete minor of order at least $c^{\prime} \sqrt{n}$.

Together with (4) and Theorem 5 this then implies the lower bound of Theorem 1 for $r=3$. The lower bound for $r>3$ follows from Theorem 9.36 in [11] which states that for each $s \geq 3$ an increasing property that holds a.a.s. for $G(n, s)$ also holds a.a.s. for $G(n, s+1)$. 


\section{Notation AND LARGE DEVIATION INEQUALities}

5.1. Notation. Given a graph $G$ and two disjoint sets $A$ and $B$ of vertices, we say that an edge of $G$ is an $A-B$ edge if it joins a vertex in $A$ to a vertex in $B$. Given disjoint subgraphs $H$ and $H^{\prime}$ of $G$, we define $H$ - $H^{\prime}$ edges of $G$ similarly. Given $a, b \in \mathbb{R}$ we write $[a \pm b]$ for the interval $[a-b, a+b]$. We will write $\ln ^{2} n$ for $(\ln n)^{2}$. We omit floors and ceilings whenever this does not affect the argument.

5.2. A concentration inequality. In this subsection, we will state a concentration inequality which we will use several times during the proof of Theorem 6. This is Theorem 7.4 in [18. We first describe the more general setting to which this theorem applies.

Let $W$ be a finite probability space that is also a metric space with its metric denoted by $d$. Suppose that $F_{0}, \ldots, F_{s}$ is a sequence of partitions of $W$ such that $F_{j+1}$ refines $F_{j}, F_{0}$ is the partition consisting of only one part (i.e. $F_{0}=\{W\}$ ) and $F_{s}$ is the partition where each part is a single element of $W$. Suppose that whenever $A, B \in F_{j+1}$ and $C \in F_{j}$ are such that $A, B \subseteq C$, then there is a bijection $\phi: A \rightarrow B$ such that $d(x, \phi(x)) \leq c$. Now, let $w \in W$ be chosen uniformly at random and let $f: W \rightarrow \mathbb{R}$ be a function on $W$ satisfying $|f(x)-f(y)| \leq d(x, y)$. Then for all $a>0$

$$
\mathbb{P}(|f(w)-\mathbb{E}(f(w))|>a) \leq 2 \exp \left(\frac{-2 a^{2}}{s c^{2}}\right) .
$$

5.3. The hypergeometric distribution. Let $Z$ be a non-empty finite set and $Z^{\prime} \subseteq Z$. Assume that we sample a set $Y$ uniformly at random among all subsets of $Z$ having size $y$. Recall that the size of $Y \cap Z^{\prime}$ is a random variable whose distribution is hypergeometric and whose expected value is $\lambda:=y\left|Z^{\prime}\right| /|Z|$. We will often use the following concentration inequality that follows e.g. from Theorem 2.10 and Inequalities (2.5) and (2.6) in [11]:

$$
\mathbb{P}\left(|| Y \cap Z^{\prime}|-\lambda| \geq a\right) \leq 2 \exp \left(-\frac{a^{2}}{2(\lambda+a / 3)}\right)
$$

for all $a \geq 0$.

\section{Proof of Theorem 6}

6.1. Setup. Let $V_{n}$ be a set of $n$ vertices. We will expose the random multigraph $H(n)+$ $G(n, 1)$ on $V_{n}$ in stages starting with the Hamilton cycle $H(n)$. We split $H(n)$ into two paths $P_{1}, P_{2}$ of equal lengths each having $n / 2$ vertices. As described in Section 3 , the (candidate) branch sets for our minor will be subpaths of $P_{1}$ and we will use the edges of the random perfect matching $G(n, 1)$ as well as subpaths of $P_{2}$ to join them. Let us now turn to $G(n, 1)$. So consider a perfect matching $M^{*}$ on $V_{n}$ chosen uniformly at random. Our first aim is to estimate the number of $P_{1}-P_{2}$ edges of $M^{*}$.

Lemma 7. With probability $1-O\left(1 / \ln ^{2} n\right)$ the number of $P_{1}-P_{2}$ edges of $M^{*}$ lies in the interval $[n / 4 \pm \sqrt{n} \ln n]$.

Proof. This is a simple application of Chebyshev's inequality. For each vertex $v \in V\left(P_{1}\right)$ set $X_{v}:=1$ if $M^{*}$ matches $v$ to a vertex of $P_{2}$ and set $X_{v}:=0$ otherwise. Then $X:=$ $\sum_{v \in V\left(P_{1}\right)} X_{v}$ is the number of $P_{1}-P_{2}$ edges of $M^{*}$. Note that for every $v$ we have

$$
\mathbb{P}\left(X_{v}=1\right)=\frac{n / 2}{n-1}=1 / 2+O(1 / n) .
$$


So $\mathbb{E} X=(n / 2)(1 / 2+O(1 / n))=n / 4+O(1)$. Also, for distinct $v, w \in V\left(P_{1}\right)$ we have

$$
\mathbb{P}\left(X_{v}=1 \mid X_{w}=1\right)=\frac{n / 2-1}{n-3}=1 / 2+O(1 / n) .
$$

This implies that

$$
\begin{aligned}
\mathbb{E}\left(X^{2}\right) & =\sum_{v \in V\left(P_{1}\right)} \mathbb{P}\left(X_{v}=1\right)+\sum_{v \neq w \in V\left(P_{1}\right)} \mathbb{P}\left(X_{v}=X_{w}=1\right) \\
& =\mathbb{E} X+\frac{n}{2}\left(\frac{n}{2}-1\right)\left(\frac{1}{4}+O(1 / n)\right)=\frac{n^{2}}{16}+O(n)=(\mathbb{E} X)^{2}(1+O(1 / n)) .
\end{aligned}
$$

So Chebyshev's inequality implies that

$$
\mathbb{P}(|X-n / 4| \geq \sqrt{n} \ln n) \leq \mathbb{P}(|X-\mathbb{E} X| \geq(\sqrt{n} \ln n) / 2)=\frac{O(1 / n)(\mathbb{E} X)^{2}}{n \ln ^{2} n}=O\left(1 / \ln ^{2} n\right),
$$

as required.

Fix a positive constant $\varepsilon$. Throughout the proof we will assume that $\varepsilon$ is sufficiently small for our estimates to hold. (All conditions on $\varepsilon$ will involve only absolute constants, i.e. will be independent of $n$.) Suppose that $n$ is sufficiently large compared to $1 / \varepsilon$. Let $k$ and $t$ be integers such that

$$
\left(\begin{array}{l}
k \\
2
\end{array}\right)=\varepsilon^{4} n \text { and } t:=\frac{\sqrt{n}}{\varepsilon} .
$$

So $k=(1+o(1)) \varepsilon^{2} \sqrt{2 n}$. Consider any $X_{1} \subseteq V\left(P_{1}\right)$ and $X_{2} \subseteq V\left(P_{2}\right)$ such that $\left|X_{1}\right|=\left|X_{2}\right| \in$ $[n / 4 \pm \sqrt{n} \ln n]$. Let $X_{1}^{\prime} \subseteq X_{1}$ be the set of the first $k t$ vertices on $P_{1}$ in $X_{1}$. Let $X_{2}^{\prime} \subseteq X_{2}$ be any subset of size $k t$. Let $\mathcal{X}$ denote the event that $X_{1}$ and $X_{2}$ are the set of endvertices of the $P_{1}-P_{2}$ edges in our random perfect matching $M^{*}$ on $V_{n}$. Similarly, let $\mathcal{X}^{\prime}$ be the event that $M^{*}$ matches $X_{1}^{\prime}$ to $X_{2}^{\prime}$. In what follows, we will condition on both $\mathcal{X}$ and $\mathcal{X}^{\prime}$. All our probability bounds will hold regardless of what the sets $X_{1}, X_{2}, X_{1}^{\prime}, X_{2}^{\prime}$ actually are (provided that $\left|X_{1}\right|=\left|X_{2}\right|$ is in the specified range).

Pick $k$ consecutive disjoint subpaths $B_{1}, \ldots, B_{k}$ of $P_{1}$ such that $\left|V\left(B_{i}\right) \cap X_{1}\right|=\mid V\left(B_{i}\right) \cap$ $X_{1}^{\prime} \mid=t$ for all $i=1, \ldots, k$. The $B_{i}$ 's will be called candidate branch sets and the vertices in $V\left(B_{i}\right) \cap X_{1}^{\prime}$ will be called the effective vertices of $B_{i}$. We will show that a.a.s. there is a complete minor whose branch sets are almost all the $B_{i}$ 's. Set

$$
i_{0}:=\left(\log _{3} n\right) / 6 \text {. }
$$

Choose consecutive disjoint subpaths $Q_{1}, \ldots, Q_{i_{0}}$ of $P_{2}$ such that

$$
\left|Q_{i}\right|_{\mathrm{eff}}:=\left|V\left(Q_{i}\right) \cap X_{2}^{\prime}\right|=\frac{\left|X_{2}^{\prime}\right|}{3^{i}}=\frac{k t}{3^{i}}=\frac{(1+o(1)) \sqrt{2} \varepsilon n}{3^{i}} .
$$

The vertices in $V\left(Q_{i}\right) \cap X_{2}^{\prime}$ are the effective vertices of $Q_{i}$ and $\left|Q_{i}\right|_{\text {eff }}$ is the effective length of $Q_{i}$. We further divide each $Q_{i}$ into a set $\mathcal{P}_{i}$ of consecutive disjoint subpaths, each of effective length

$$
\ell_{i}:=100 \cdot 3^{i-1} \text {. }
$$

(So each of these subpaths meets $X_{2}^{\prime}$ in precisely $\ell_{i}$ vertices.) Note that

$$
\ell_{i_{0}} \leq 100 \cdot 3^{i_{0}}=100 n^{1 / 6}
$$

and

$$
\left|\mathcal{P}_{i}\right|=\frac{\left|Q_{i}\right|_{\mathrm{eff}}}{\ell_{i}}=\frac{k t}{300 \cdot 9^{i-1}}
$$


Thus $\left|\mathcal{P}_{i_{0}}\right|=\Theta\left(n^{2 / 3}\right)$. The strategy of our proof is to expose the neighbours of the (effective) vertices from $X_{2}^{\prime}$ in our random perfect matching $M^{*}$ in $i_{0}$ stages. More precisely, during the $i$ th stage we will expose the neighbours of the effective vertices in $Q_{i}$ (for every $1 \leq i \leq i_{0}$ ). We will show that with high probability during each stage we can use the paths in $\mathcal{P}_{i}$ to join a large proportion of all those pairs of candidate branch sets that are still unjoined after the previous stages. More precisely, an unjoined pair $\left(B, B^{\prime}\right)$ of candidate branch sets can be joined through $P \in \mathcal{P}_{i}$ if our random perfect matching $M^{*}$ contains both a $B-P$ edge and a $B^{\prime}-P$ edge. In this case we will say that $P$ can be used to join the pair $\left(B, B^{\prime}\right)$. Of course, if we use $P$ to join $\left(B, B^{\prime}\right)$ then $P$ cannot be used to join another unjoined pair of candidate branch sets.

Let us make the above more precise. Given $1 \leq i \leq i_{0}$, let $\mathcal{U}_{i-1}$ denote the set of pairs of candidate branch sets that are still unjoined after the $(i-1)$ th stage. So $\mathcal{U}_{0}$ is the set of all pairs of candidate branch sets. Note that

$$
U_{0}:=\left|\mathcal{U}_{0}\right|=\left(\begin{array}{l}
k \\
2
\end{array}\right)=\varepsilon^{4} n .
$$

We will show that with high probability during the $i$ th stage we can join $26\left|\mathcal{U}_{i-1}\right| / 27$ pairs in $\mathcal{U}_{i-1}$ using the paths belonging to $\mathcal{P}_{i}$. So inductively we will prove that with high probability

$$
U_{i}:=\left|\mathcal{U}_{i}\right|=\frac{U_{0}}{27^{i}}=\frac{\varepsilon^{4} n}{27^{i}} .
$$

Suppose that (14) holds for all $j<i$ and that we now wish to analyze the $i$ th stage. It will turn out that the pairs in $\mathcal{U}_{i-1}$ which contain candidate branch sets lying in too many other pairs from $\mathcal{U}_{i-1}$ create problems. So we will ignore these pairs. More precisely, let $\mathcal{B}_{i-1}$ be the set of all those candidate branch sets that belong to more than

$$
\Delta_{i-1}:=\frac{(3 / 2)^{i-1} U_{i-1}}{\varepsilon^{1 / 8} k}=\frac{U_{0}}{\varepsilon^{1 / 8}(2 \cdot 9)^{i-1} k}
$$

pairs in $\mathcal{U}_{i-1}$. Note that since $\left|\mathcal{B}_{i-1}\right| \Delta_{i-1} \leq 2 U_{i-1}$ we have

$$
\left|\mathcal{B}_{i-1}\right| \leq \frac{2 U_{i-1}}{\Delta_{i-1}} \leq \frac{2 \varepsilon^{1 / 8} k}{(3 / 2)^{i-1}}
$$

Let $\mathcal{U}_{i-1}^{*}$ be the set of all those pairs in $\mathcal{U}_{i-1}$ having at least one branch set in $\mathcal{B}_{i-1}$. Call these pairs bad. If $\left|\mathcal{U}_{i-1}^{*}\right| \geq 26 U_{i-1} / 27$, delete precisely $26 U_{i-1} / 27$ bad pairs from $\mathcal{U}_{i-1}$ to obtain $\mathcal{U}_{i}$. If $\left|\mathcal{U}_{i-1}^{*}\right|<26 U_{i-1} / 27$ we let $\mathcal{U}_{i-1}^{\prime}:=\mathcal{U}_{i-1} \backslash \mathcal{U}_{i-1}^{*}$. We will show that during the $i$ th stage with high probability we can join all but $U_{i-1} / 27$ pairs in $\mathcal{U}_{i-1}^{\prime}$. We let $\mathcal{U}_{i}$ be the set of the remaining unjoined pairs in $\mathcal{U}_{i-1}^{\prime}$. Thus in both cases $U_{i}=\left|\mathcal{U}_{i}\right|$ satisfies (14) with high probability.

After the end of stage $i_{0}$ will delete all the candidate branch sets in $\mathcal{B}_{0} \cup \cdots \cup B_{i_{0}-1}$ (see Section 6.5). The number of these candidate branch sets is

$$
\sum_{i=1}^{i_{0}}\left|\mathcal{B}_{i-1}\right| \stackrel{\sqrt[16]{\leq}}{\leq} \sum_{i \geq 1} \frac{2 \varepsilon^{1 / 8} k}{(3 / 2)^{i-1}}=6 \varepsilon^{1 / 8} k .
$$

6.2. Bounds on the number of effective vertices still available. We will now estimate the number of all those effective vertices in each candidate branch set that are joined to a path in $\mathcal{P}_{1} \cup \cdots \cup \mathcal{P}_{i-1}$, i.e. that are matched after the first $i-1$ stages. The total number of 
effective vertices in the candidate branch sets that are matched after the first $i-1$ stages is

$$
\sum_{j \leq i-1}\left|Q_{j}\right|_{\mathrm{eff}} \stackrel{\underline{\underline{9}}}{=} k t \sum_{j=1}^{i-1} \frac{1}{3^{j}}=\frac{k t}{2}\left(1-3^{-(i-1)}\right)=: x_{i-1} .
$$

Each $x_{i-1}$-subset of the union $X_{1}^{\prime}$ of all the effective vertices in the candidate branch sets is equally likely to be the set of these matched vertices. Thus for every candidate branch set $B$ the distribution of the number $\operatorname{eff}_{i}^{\prime}(B)$ of all those effective vertices in $B$ which are matched to (effective vertices on) paths in $\mathcal{P}_{1} \cup \cdots \cup \mathcal{P}_{i-1}$ is hypergeometric. Since in total $B$ contains $t$ effective vertices and $\left|X_{1}^{\prime}\right|=k t$ we can now use (무) to see that

$$
\mathbb{P}\left(\left|\operatorname{eff}_{i}^{\prime}(B)-x_{i-1} t / k t\right| \geq n^{1 / 4} \ln n \mid \mathcal{X}, \mathcal{X}^{\prime}\right) \leq \exp \left(-\Omega\left(\ln ^{2} n\right)\right) .
$$

Thus,

$$
\operatorname{eff}_{i}^{\prime}(B) \in\left[\frac{x_{i-1}}{k} \pm n^{1 / 4} \ln n\right] \subseteq\left[\frac{t}{2}\left(1-3^{-(i-1)}\right) \pm n^{1 / 3}\right]
$$

with (conditional) probability $1-\exp \left(-\Omega\left(\ln ^{2} n\right)\right)$. Now, let $\operatorname{eff}_{i}(B):=t-\operatorname{eff}_{i}^{\prime}(B)$ be the number of all those effective vertices in $B$ that are still unmatched after the first $i-1$ stages and let $\operatorname{Eff}_{i}(B)$ denote the set of all those effective vertices. Thus with (conditional) probability $1-k \exp \left(-\Omega\left(\ln ^{2} n\right)\right)=1-\exp \left(-\Omega\left(\ln ^{2} n\right)\right)$ we have

$$
\operatorname{eff}_{i}(B) \in\left[\frac{t}{2}\left(1+3^{-(i-1)}\right) \pm n^{1 / 3}\right]
$$

for all candidate branch sets $B$.

Let $M_{i-1}^{*}$ be any matching which matches the set $\operatorname{Eff}\left(Q_{1}\right) \cup \cdots \cup \operatorname{Eff}\left(Q_{i-1}\right)$ of effective vertices on the paths $Q_{1}, \ldots, Q_{i-1}$ (equivalently the set of effective vertices on the paths in $\mathcal{P}_{1} \cup \cdots \cup \mathcal{P}_{i-1}$ ) into the set of effective vertices in the candidate branch sets. Suppose that $M_{i-1}^{*}$ is the submatching of our random matching $M^{*}$ exposed after the first $i-1$ stages. Then $M_{i-1}^{*}$ determines $\operatorname{Eff}_{i}(B)$ for every candidate branch set $B$. Moreover, by considering a fixed ordering of all the pairs in $\mathcal{U}_{0}$, we may assume that $M_{i-1}^{*}$ also determines $\mathcal{U}_{i-1}$. Call $M_{i-1}^{*}$ good if (19) holds for all candidate branch sets $B$ and if (14) holds for $i-1$. Consider any good $M_{i-1}^{*}$ and let $\mathcal{M}_{i-1}^{*}$ denote the event that $M_{i-1}^{*}$ is the submatching of our random matching $M^{*}$ exposed after the first $i-1$ stages. From now on we will condition on $\mathcal{X}$, $\mathcal{X}^{\prime}$ and $\mathcal{M}_{i-1}^{*}$ and we let $\mathbb{P}_{i}(\cdot)$ denote the corresponding conditional probability measure that arises from choosing a random matching from the set $\operatorname{Eff}\left(Q_{i}\right)$ of effective vertices on $Q_{i}$ into the set $\bigcup_{j=1}^{k} \operatorname{Eff}_{i}\left(B_{j}\right)$ of all those effective vertices in the candidate branch sets which are not already endvertices of edges in $M_{i-1}^{*}$ (i.e. into the set of all those effective vertices in the candidate branch sets that are still unmatched after the first $i-1$ stages).

Given $\mathcal{S} \subseteq \mathcal{P}_{i}$ and a candidate branch set $B$, we let Eff $_{\mathcal{S}}(B)$ denote the set of all those effective vertices in $B$ that are matched to some (effective) vertex on a path in $\mathcal{S}$ (in our random matching $\left.M^{*}\right)$. Assume that $|\mathcal{S}|=\alpha\left|\mathcal{P}_{i}\right|$ where $1 / 2 \leq \alpha \leq 1$. Let

$$
I(\alpha):=\left[\frac{\alpha t}{3^{i}}\left(1 \pm \frac{1}{4}\right)\right]
$$

and let $\mathcal{E}_{\mathcal{S}}$ denote the event that $\left|\operatorname{Eff}_{\mathcal{S}}(B)\right| \in I(\alpha)$ for every candidate branch set $B$. Let $\overline{\mathcal{E}_{\mathcal{S}}}$ denote the complement of $\mathcal{E}_{\mathcal{S}}$.

Lemma 8. $\mathbb{P}_{i}\left(\overline{\mathcal{E}_{\mathcal{S}}}\right)=\exp \left(-\Omega\left(\ln ^{2} n\right)\right)$. 
Proof. Consider any candidate branch set $B$. Note that $\left|\operatorname{Eff}_{\mathcal{S}}(B)\right|$ is hypergeometrically distributed with mean $\lambda:=\operatorname{eff}_{i}(B)|\mathcal{S}| \ell_{i} /\left(k t-x_{i-1}\right)$. But $|\mathcal{S}| \ell_{i}=\alpha\left|\mathcal{P}_{i}\right| \ell_{i}=\alpha\left|Q_{i}\right|_{\text {eff }}$ and $k t-x_{i-1}=\frac{k t}{2}\left(1+3^{-(i-1)}\right)$ by (18). So (19) implies that

$$
\frac{\operatorname{eff}_{i}(B)}{k t-x_{i-1}} \in\left[\frac{\frac{t}{2}\left(1+3^{-(i-1)}\right) \pm n^{1 / 3}}{\frac{k t}{2}\left(1+3^{-(i-1)}\right)}\right] \subseteq\left[\frac{1}{k}\left(1 \pm \frac{1}{5}\right)\right]
$$

and thus

$$
\lambda \in\left[\alpha\left|Q_{i}\right|_{\mathrm{eff}}\left(\frac{1}{k}\left(1 \pm \frac{1}{5}\right)\right)\right] \stackrel{\underline{9}}{=}\left[\frac{\alpha t}{3^{i}}\left(1 \pm \frac{1}{5}\right)\right] .
$$

In particular, together with (6) this implies that

$$
\mathbb{P}_{i}\left(|| \operatorname{Eff}_{\mathcal{S}}(B)|-\lambda| \geq n^{1 / 4} \ln n\right)=\exp \left(-\Omega\left(\ln ^{2} n\right)\right) .
$$

So with probability at most $k \exp \left(-\Omega\left(\ln ^{2} n\right)\right)=\exp \left(-\Omega\left(\ln ^{2} n\right)\right)$ there is a candidate branch set $B$ with

as required.

$$
\left|\operatorname{Eff}_{\mathcal{S}}(B)\right| \notin\left[\frac{\alpha t}{3^{i}}\left(1 \pm \frac{1}{5}\right) \pm n^{1 / 4} \ln n\right] \stackrel{\sqrt[76]{(20}}{\subseteq} I(\alpha),
$$

6.3. A lower bound for the degrees of the pairs of candidate branch sets in $\mathcal{G}_{i}$. Recall that, as described in the paragraph after (16), when analyzing the $i$ th stage, we may assume that $\left|\mathcal{U}_{i-1}^{*}\right|<26 U_{i-1} / 27$ and thus $\mathcal{U}_{i-1}^{\prime}$ is well defined. Given a candidate branch set $B$ and path $P \in \mathcal{P}_{i}$, we write $P \sim B$ if some effective vertex on $P$ is matched to some vertex in $\operatorname{Eff}_{i}(B)$ (in our random matching $M^{*}$ ). Consider an auxiliary bipartite graph $\mathcal{G}_{i}$ whose vertex classes are $\mathcal{U}_{i-1}^{\prime}$ and $\mathcal{P}_{i}$ and in which a pair $\left(B, B^{\prime}\right) \in \mathcal{U}_{i-1}^{\prime}$ is adjacent to $P \in \mathcal{P}_{i}$ if $P$ can be used to join $\left(B, B^{\prime}\right)$, i.e. if $P \sim B$ and $P \sim B^{\prime}$. We will now estimate the degrees of the vertices in $\mathcal{U}_{i-1}^{\prime}$ in $\mathcal{G}_{i}$. Given $\mathcal{S} \subseteq \mathcal{P}_{i}$, we let $d_{\mathcal{G}_{i}}(L, \mathcal{S})$ denote the degree of a vertex/pair $L \in \mathcal{U}_{i-1}^{\prime}$ into the set $\mathcal{S}\left(\right.$ in $\left.\mathcal{G}_{i}\right)$.

Lemma 9. Suppose that $1 / 2 \leq \alpha \leq 1$ (where $\alpha$ may depend on $n$ ). Fix $L \in \mathcal{U}_{i-1}^{\prime}$ and $\mathcal{S} \subseteq \mathcal{P}_{i}$ with $|\mathcal{S}|=\alpha\left|\mathcal{P}_{i}\right|$. Then $\mathbb{P}_{i}\left(d_{\mathcal{G}_{i}}(L, \mathcal{S}) \leq 1 /\left(2 \varepsilon^{3}\right)\right)<3 \varepsilon$.

Proof. Let $L=\left(B, B^{\prime}\right)$. Our aim is to show that

$$
\mathbb{P}_{i}\left(d_{\mathcal{G}_{i}}(L, \mathcal{S}) \leq 1 /\left(2 \varepsilon^{3}\right) \mid \mathcal{E}_{\mathcal{S}}\right)<2 \varepsilon
$$

This implies the lemma since

$$
\begin{aligned}
& \mathbb{P}_{i}\left(d_{\mathcal{G}_{i}}(L, \mathcal{S}) \leq 1 /\left(2 \varepsilon^{3}\right)\right) \leq \mathbb{P}_{i}\left(d_{\mathcal{G}_{i}}(L, \mathcal{S}) \leq 1 /\left(2 \varepsilon^{3}\right) \mid \mathcal{E}_{\mathcal{S}}\right)+\mathbb{P}_{i}\left(\overline{\mathcal{E}_{\mathcal{S}}}\right) \\
& \text { 211, Lemma } 8 \\
& \leq \quad 2 \varepsilon+\exp \left(-\Omega\left(\ln ^{2} n\right)\right)<3 \varepsilon \text {. }
\end{aligned}
$$

To estimate the number of all those paths in $\mathcal{S}$ that are neighbours of both $B$ and $B^{\prime}$ in the auxiliary graph $\mathcal{G}_{i}$, we will first bound the number of paths in $\mathcal{S}$ that are neighbours of $B$ and then we will estimate how many of them are neighbours of $B^{\prime}$. More precisely, we will first show that most of the paths $P \in \mathcal{S}$ with $P \sim B$ are joined to $B$ by exactly one (matching) edge. Let us condition first on a particular realization $E_{B}$ of $\operatorname{Eff}_{\mathcal{S}}(B)$ with $\left|E_{B}\right| \in I(\alpha)$. Denote the corresponding probability subspace of $\mathbb{P}_{i}$ (where we condition on the event that $\operatorname{Eff}_{\mathcal{S}}(B)=E_{B}$ and on $\mathcal{E}_{\mathcal{S}}$ ) by $\mathbb{P}_{i, \mathcal{E}_{\mathcal{S}}, E_{B}}$. Assuming an arbitrary ordering of the vertices in $E_{B}$, we expose their neighbours (in the random matching) on the paths in $\mathcal{S}$ one by one according to this ordering. We say that the $j$ th vertex fails if its neighbour lies in a path from $\mathcal{S}$ that already contains a neighbour of the previously exposed vertices. Note that 
the number of paths in $\mathcal{S}$ containing more than one neighbour of $E_{B}$ is bounded above by the number of failures that occur during the exposure of the neighbours of $E_{B}$. Suppose we have exposed the neighbours of the first $j-1$ vertices in $E_{B}$. Let the corresponding event be $\mathcal{C}_{j-1}$. To estimate the probability that the $j$ th vertex fails, observe that the number of all those paths in $\mathcal{S}$ that already have a neighbour in $E_{B}$ is less than $j$ and each of them contains less than $\ell_{i}$ effective vertices which are still available. Note that this holds regardless of what $\mathcal{C}_{j-1}$ is. Thus

$\mathbb{P}_{i, \mathcal{E}_{\mathcal{S}}, E_{B}}$ (the $j$ th vertex fails $\left.\mid \mathcal{C}_{j-1}\right)<\frac{j \ell_{i}}{\alpha\left|\mathcal{P}_{i}\right| \ell_{i}-(j-1)} \leq \frac{\left|E_{B}\right| \ell_{i}}{\alpha\left|\mathcal{P}_{i}\right| \ell_{i}-\left|E_{B}\right|} \stackrel{\sqrt[8]{6} \text {, (12) }}{\leq} \frac{2\left|E_{B}\right|}{\alpha\left|\mathcal{P}_{i}\right|}=: \tilde{p}$.

In particular, let $\mathcal{D}_{j-1}$ be any event which depends only on the neighbours of the first $j-1$ vertices in $E_{B}$. Then

$$
\left.\mathbb{P}_{i, \mathcal{E}_{\mathcal{S}}, E_{B}} \text { (the } j \text { th vertex fails } \mid \mathcal{D}_{j-1}\right) \leq \tilde{p} .
$$

Now let $A=\left\{a_{1}, \ldots, a_{r}\right\}$ be any set of vertices in $E_{B}$ (where $a_{q}$ precedes $a_{q+1}$ in the ordering of $E_{B}$ ) and let Fail $_{A}$ denote the event that the set of failure vertices equals $A$. Then

$$
\mathbb{P}_{i, \mathcal{E}_{\mathcal{S}}, E_{B}}\left(\text { Fail }_{A}\right) \leq \prod_{q=1}^{r} \mathbb{P}_{i, \mathcal{E}_{\mathcal{S}}, E_{B}}\left(a_{q} \text { fails } \mid a_{1}, \ldots, a_{q-1} \text { fail } \stackrel{\stackrel{22}{\leq}}{\leq} \tilde{p}^{r} .\right.
$$

This in turn implies that

$$
\mathbb{P}_{i, \mathcal{E}_{\mathcal{S}}, E_{B}}(\geq f \text { failures }) \leq \sum_{r=f}^{\left|E_{B}\right|} \sum_{\substack{A \subseteq E_{B} \\
|A|=r}} \mathbb{P}_{i, \mathcal{E}_{\mathcal{S}}, E_{B}}\left(\text { Fail }_{A}\right) \leq \sum_{r=f}^{\left|E_{B}\right|}\left(\begin{array}{c}
\left|E_{B}\right| \\
r
\end{array}\right) \tilde{p}^{r} \leq \sum_{r=f}^{\left|E_{B}\right|}\left(\frac{e\left|E_{B}\right|}{r} \frac{2\left|E_{B}\right|}{\alpha\left|\mathcal{P}_{i}\right|}\right)^{r}
$$

Since $\left|E_{B}\right| \in I(\alpha)$ we have

$$
\frac{\left|E_{B}\right|^{2}}{\alpha\left|\mathcal{P}_{i}\right|} \stackrel{12}{\in}, \underset{\epsilon}{\in}\left[(1 \pm 1 / 4)^{2} \frac{\alpha^{2} t^{2}}{9^{i}} \frac{1}{\alpha} \frac{300 \cdot 9^{i}}{9 k t}\right] \stackrel{\sqrt[7]{\complement}}{\subseteq}\left[(1 \pm 2 / 3) \frac{100 \alpha}{3 \sqrt{2} \varepsilon^{3}}\right] .
$$

Let $\mathcal{F}_{B}$ denote the event that at least $1000 / \varepsilon^{3}$ failures occur when we expose the neighbours of the vertices in $E_{B}$. Thus by setting $f:=1000 / \varepsilon^{3}$, we obtain

$$
\mathbb{P}_{i, \mathcal{E}_{\mathcal{S}}, E_{B}}\left(\mathcal{F}_{B}\right)=\mathbb{P}_{i, \mathcal{E}_{\mathcal{S}}, E_{B}}(\geq f \text { failures }) \leq \sum_{r \geq f}\left(\frac{5 \sqrt{2} \cdot 100 e \alpha}{9 \varepsilon^{3} r}\right)^{r} \leq \sum_{r \geq f}(1 / 2)^{r} \leq \varepsilon .
$$

Let $\overline{\mathcal{F}_{B}}$ denote the complement of $\mathcal{F}_{B}$. Note that if $\overline{\mathcal{F}_{B}}$ occurs, then there are at least $\left|E_{B}\right|-1000 / \varepsilon^{3}$ paths in $\mathcal{S}$ that are joined to $E_{B} \subseteq B$ by exactly one (matching) edge. Let $\mathcal{S}(B)$ denote the set of these paths. So

$$
\left|E_{B}\right|-1000 / \varepsilon^{3} \leq|\mathcal{S}(B)| \leq\left|E_{B}\right|
$$

(for the second inequality we need not assume that $\overline{\mathcal{F}_{B}}$ holds). Now we additionally condition on a specific realization $E_{B^{\prime}}$ of $\operatorname{Eff}_{\mathcal{S}}\left(B^{\prime}\right)$ with $\left|E_{B^{\prime}}\right| \in I(\alpha)$. As above, we fix an arbitrary ordering on the vertices in $E_{B^{\prime}}$ according to which we expose their neighbours in $\mathcal{S}$. We say that the $j$ th vertex of $E_{B^{\prime}}$ is useful if it is adjacent to a vertex lying on a path from $\mathcal{S}(B)$ such that none of the previous vertices in $E_{B^{\prime}}$ is joined to this path. Note that if $U\left(B^{\prime}\right)$ denotes the set of vertices in $E_{B^{\prime}}$ that are useful, then

$$
\left|U\left(B^{\prime}\right)\right| \leq d_{\mathcal{G}_{i}}(L, \mathcal{S})
$$

Given $\overline{\mathcal{F}_{B}}$, we will show that with high probability $\left|U\left(B^{\prime}\right)\right| \geq 1 /\left(2 \varepsilon^{3}\right)$.

Note that there are exactly $R:=\alpha\left|\mathcal{P}_{i}\right| \ell_{i}-\left|E_{B}\right|$ effective vertices on the paths in $\mathcal{S}$ that are still available to be matched to the vertices of $E_{B^{\prime}}$. Put $s:=|\mathcal{S}(B)|\left(\ell_{i}-1\right)$ and let $C$ 
be any subset of $E_{B^{\prime}}$ with $c:=|C| \leq 1 /\left(2 \varepsilon^{3}\right)$. Suppose that $C$ is the set of useful vertices in $E_{B^{\prime}}$. Then the vertices in $C$ are matched to effective vertices on different paths in $\mathcal{S}(B)$. So there are $|\mathcal{S}(B)|_{c}\left(\ell_{i}-1\right)^{c} \leq s^{c}$ choices for the neighbours of $C$. Moreover, each vertex $x \in E_{B^{\prime}} \backslash C$ is either matched to an effective vertex on a path in $\mathcal{S}(B)$ which already contains a neighbour of $C$ or $x$ is matched to an effective vertex on a path in $\mathcal{S} \backslash \mathcal{S}(B)$. There are less than $c \ell_{i}$ choices for a neighbour of $x$ having the first property and $R-s$ choices for a neighbour of $x$ having the second property. Thus in total the number of choices for the neighbours of $E_{B^{\prime}} \backslash C$ is at most

$$
\begin{aligned}
\sum_{q=0}^{\left|E_{B^{\prime}}\right|-c}\left(\left|E_{B^{\prime}}\right|-c\right)_{q}\left(c \ell_{i}\right)^{q}(R-s)_{\left|E_{B^{\prime}}\right|-c-q} & \leq(R-s)_{\left|E_{B^{\prime}}\right|-c} \sum_{q=0}^{\left|E_{B^{\prime}}\right|-c}\left(\frac{c \ell_{i}\left|E_{B^{\prime}}\right|}{R / 2}\right)^{q} \\
& \leq(R-s)_{\left|E_{B^{\prime}}\right|-c} \sum_{q \geq 0}\left(\frac{1}{2}\right)^{q}=2(R-s)_{\left|E_{B^{\prime}}\right|-c} .
\end{aligned}
$$

(Here we used that $\left|E_{B^{\prime}}\right|=O(\sqrt{n})=o\left(\left|\mathcal{P}_{i}\right|\right)$ and so $s=o(R)$ as well as $\left|E_{B^{\prime}}\right| \ell_{i}=o(R)$.) Setting $p:=s / R$ we obtain

$$
\begin{aligned}
\mathbb{P}_{i, \mathcal{E}_{\mathcal{S}}, E_{B}} & \left(U\left(B^{\prime}\right)=C \mid \overline{\mathcal{F}_{B}}, \operatorname{Eff}_{\mathcal{S}}\left(B^{\prime}\right)=E_{B^{\prime}}\right) \leq \frac{2 s^{c}(R-s)_{\left|E_{B^{\prime}}\right|-c}}{(R)_{\left|E_{B^{\prime}}\right|}} \\
& \leq 2 s^{c}\left(\frac{R-s}{R}\right)^{\left|E_{B^{\prime}}\right|-c}\left(\frac{1}{R-\left|E_{B^{\prime}}\right|}\right)^{c}=2\left(\frac{s}{R-\left|E_{B^{\prime}}\right|}\right)^{c}\left(1-\frac{s}{R}\right)^{\left|E_{B^{\prime}}\right|-c} \\
& \leq 2\left(\frac{10}{9}\right)^{c}\left(\frac{s}{R}\right)^{c}\left(1-\frac{s}{R}\right)^{\left|E_{B^{\prime}}\right|-c}=2\left(\frac{10}{9}\right)^{c} p^{c}(1-p)^{\left|E_{B^{\prime}}\right|-c} .
\end{aligned}
$$

(In the second inequality we used that $\frac{a-j}{b-j}<\frac{a}{b}$, for $0<j<a<b$ and in the last inequality we again used that $\left|E_{B^{\prime}}\right|=o(R)$.) Thus

$$
\begin{aligned}
\mathbb{P}_{i, \mathcal{E}_{\mathcal{S}}, E_{B}}\left(\left|U\left(B^{\prime}\right)\right| \leq 1 /\left(2 \varepsilon^{3}\right) \mid\right. & \left.\overline{\mathcal{F}_{B}}, \operatorname{Eff}_{\mathcal{S}}\left(B^{\prime}\right)=E_{B^{\prime}}\right) \\
& \leq 2(10 / 9)^{1 /\left(2 \varepsilon^{3}\right)} \sum_{c \leq 1 /\left(2 \varepsilon^{3}\right)}\left(\begin{array}{c}
\left|E_{B^{\prime}}\right| \\
c
\end{array}\right) p^{c}(1-p)^{\left|E_{B^{\prime}}\right|-c} .
\end{aligned}
$$

Observe that the sum on the right-hand side is the probability that a binomial random variable $Y$ with parameters $\left|E_{B^{\prime}}\right|, p$ is at most $1 /\left(2 \varepsilon^{3}\right)$. To bound this probability from above, we will use the following Chernoff bound (see e.g. Inequality (2.9) in [11]):

$$
\mathbb{P}(Y \leq \mathbb{E} Y / 2) \leq 2 \exp (-\mathbb{E} Y / 12)
$$

Note that by (25) and the definition of $R$, we have

$$
p=\frac{s}{R}=\frac{|\mathcal{S}(B)|\left(\ell_{i}-1\right)}{R} \geq \frac{\left(\left|E_{B}\right|-1000 / \varepsilon^{3}\right)\left(\ell_{i}-1\right)}{\alpha\left|\mathcal{P}_{i}\right| \ell_{i}}>\frac{\left|E_{B}\right|}{\alpha\left|\mathcal{P}_{i}\right|} \frac{98}{100},
$$

where the last inequality holds since $\ell_{i} \geq 100$. Moreover, the bound (23) also holds if we replace $\left|E_{B}\right|^{2}$ by $\left|E_{B}\right|\left|E_{B^{\prime}}\right|$. So altogether we have

$$
\mathbb{E} Y=\left|E_{B^{\prime}}\right| p \geq \frac{98}{100} \frac{\left|E_{B^{\prime}}\right|\left|E_{B}\right|}{\alpha\left|\mathcal{P}_{i}\right|} \stackrel{\sqrt{23} \mid}{\geq} \frac{2 \alpha}{\varepsilon^{3}} \geq \frac{1}{\varepsilon^{3}} .
$$

Thus (28) implies that

$$
\sum_{c \leq 1 /\left(2 \varepsilon^{3}\right)}\left(\begin{array}{c}
\left|E_{B^{\prime}}\right| \\
c
\end{array}\right) p^{c}(1-p)^{\left|E_{B^{\prime}}\right|-c} \leq 2 \exp \left(-1 /\left(12 \varepsilon^{3}\right)\right) .
$$


Substituting this bound into (27), we obtain

$\mathbb{P}_{i, \mathcal{E}_{\mathcal{S}}, E_{B}}\left(\left|U\left(B^{\prime}\right)\right| \leq 1 /\left(2 \varepsilon^{3}\right) \mid \overline{\mathcal{F}_{B}}, \operatorname{Eff}_{\mathcal{S}}\left(B^{\prime}\right)=E_{B^{\prime}}\right) \leq 4\left((10 / 9)^{6} / e\right)^{1 /\left(12 \varepsilon^{3}\right)} \leq(9 / 10)^{1 /\left(12 \varepsilon^{3}\right)} \leq \varepsilon$.

Since $E_{B^{\prime}}$ was an arbitrary realization of $\operatorname{Eff}_{\mathcal{S}}\left(B^{\prime}\right)$ with $\left|E_{B^{\prime}}\right| \in I(\alpha)$, this implies that

$$
\mathbb{P}_{i, \mathcal{E}_{\mathcal{S}}, E_{B}}\left(\left|U\left(B^{\prime}\right)\right| \leq 1 /\left(2 \varepsilon^{3}\right) \mid \overline{\mathcal{F}_{B}}\right) \leq \varepsilon
$$

and thus

$$
\begin{aligned}
\mathbb{P}_{i, \mathcal{E}_{\mathcal{S}}, E_{B}}\left(\left|U\left(B^{\prime}\right)\right| \leq 1 /\left(2 \varepsilon^{3}\right)\right) & \leq \mathbb{P}_{i, \mathcal{E}_{\mathcal{S}}, E_{B}}\left(\left|U\left(B^{\prime}\right)\right| \leq 1 /\left(2 \varepsilon^{3}\right) \mid \overline{\mathcal{F}_{B}}\right)+\mathbb{P}_{i, \mathcal{E}_{\mathcal{S}}, E_{B}}\left(\mathcal{F}_{B}\right) \\
& \stackrel{(24)}{\leq} 2 \varepsilon .
\end{aligned}
$$

Finally, since $E_{B}$ was an arbitrary realization of $\operatorname{Eff}_{\mathcal{S}}(B)$ with $\left|E_{B}\right| \in I(\alpha)$ it follows that

$$
\mathbb{P}_{i}\left(d_{\mathcal{G}_{i}}(L, \mathcal{S}) \leq 1 /\left(2 \varepsilon^{3}\right) \mid \mathcal{E}_{\mathcal{S}}\right) \stackrel{(26)}{\leq} \mathbb{P}_{i}\left(\left|U\left(B^{\prime}\right)\right| \leq 1 /\left(2 \varepsilon^{3}\right) \mid \mathcal{E}_{\mathcal{S}}\right) \stackrel{(29]}{\leq} 2 \varepsilon
$$

as required.

Now given $\mathcal{S} \subseteq \mathcal{P}_{i}$, we let $\mathcal{U}(\mathcal{S})$ be the set of all those pairs in $\mathcal{U}_{i-1}^{\prime}$ that have degree at most $1 /\left(2 \varepsilon^{3}\right)$ into $\mathcal{S}$ (in our auxiliary graph $\mathcal{G}_{i}$ ). So if $1 / 2 \leq \alpha \leq 1$ and $|\mathcal{S}|=\alpha\left|\mathcal{P}_{i}\right|$ then Lemma 9 implies that

$$
\mathbb{E}_{i}(|\mathcal{U}(\mathcal{S})|) \leq 3 \varepsilon\left|\mathcal{U}_{i-1}^{\prime}\right| \leq \frac{U_{i-1}}{2 \cdot 27}
$$

where $\mathbb{E}_{i}(\cdot)$ denotes the expectation that arises from the probability measure $\mathbb{P}_{i}(\cdot)$.

Lemma 10. Let $1 / 2 \leq \alpha \leq 1$. Then every $\mathcal{S} \subseteq \mathcal{P}_{i}$ with $|\mathcal{S}|=\alpha\left|\mathcal{P}_{i}\right|$ satisfies

$$
\mathbb{P}_{i}\left(|\mathcal{U}(\mathcal{S})|>\frac{U_{i-1}}{27}\right) \leq 2 \exp \left(-\frac{2 \varepsilon^{8} n}{\left(3^{i-1}\right)^{7}}\right)
$$

as well as

$$
\mathbb{P}_{i}\left(|\mathcal{U}(\mathcal{S})|>\frac{U_{i-1}}{27}\right) \leq 2 \exp \left(-\varepsilon^{4} 3^{(i-1) / 4}\right) .
$$

Proof. Our aim is to apply (5) to show that $|\mathcal{U}(\mathcal{S})|$ is concentrated around its expected value. We first prove (31). Here $W$ will be the space of all those matchings which match the set $\operatorname{Eff}\left(Q_{i}\right)$ of effective vertices on $Q_{i}$ into the set $\bigcup_{j=1}^{k} \operatorname{Eff}_{i}\left(B_{j}\right)$ of all those effective vertices in the candidate branch sets that are still unmatched after the first $i-1$ stages (equipped with the uniform distribution). (Recall that $\operatorname{Eff}_{i}(B)$ is fixed since we condition on $\mathcal{M}_{i-1}$.) So each matching in $W$ consists of $\left|Q_{i}\right|_{\text {eff }}$ edges. The metric $d$ on $W$ is defined by $d\left(M, M^{\prime}\right):=2 \ell_{i}\left|M \triangle M^{\prime}\right|$ for all $M, M^{\prime} \in W$. It is easy to see that this is indeed a metric.

So let us now define the partitions $F_{0}, \ldots, F_{\left|Q_{i}\right|_{\mathrm{eff}}} \cdot F_{0}:=\{W\}$ and each part of $F_{\left|Q_{i}\right|_{\text {eff }}}$ will consist of a single matching in $W$. To define $F_{j}$ for $1 \leq j<\left|Q_{i}\right|_{\text {eff }}$, fix a linear ordering on the vertices in $\operatorname{Eff}\left(Q_{i}\right)$. Given a matching $M \in W$, the $j$-prefix of $M$ is the set of all edges in $M$ adjacent to the first $j$ vertices in $\operatorname{Eff}\left(Q_{i}\right)$. Each part of the partition $F_{j}$ will consist of all those matchings in $W$ having the same $j$-prefix. Clearly $F_{j+1}$ refines $F_{j}$.

To define the bijection $\phi$, consider any two parts $A \neq B$ of $F_{j+1}$ and any part $C$ of $F_{j}$ such that $A, B \subseteq C$. So if $M \in A$ and $M^{\prime} \in B$, then $M$ and $M^{\prime}$ have the same $j$-prefix and they differ at the edge that is adjacent to the $(j+1)$ th vertex in $\operatorname{Eff}\left(Q_{i}\right)$. Let $v_{A}$ and $v_{B}$ be the neighbours of the $(j+1)$ th vertex in $M$ and $M^{\prime}$, respectively. Note that $v_{A}$ does not depend on the choice of $M \in A$ and similarly for $v_{B}$. We define $\phi: A \rightarrow B$ by saying that for all $M \in A$ the matching $\phi(M)$ is obtained from $M$ as follows: the $(j+1)$ th vertex in $\operatorname{Eff}\left(Q_{i}\right)$ is now matched to $v_{B}$ and $v_{A}$ is matched to the neighbour of $v_{B}$ in $M$, every other edge of $M$ 
remains unchanged. Thus the size of the symmetric difference of $M$ and $\phi(M)$ is 4 and so $d(M, \phi(M)) \leq 8 \ell_{i}$. So we can take $c:=8 \ell_{i}$.

Now note that $|\mathcal{U}(\mathcal{S})|$ is a function whose value is determined by a matching from $W$ chosen uniformly at random. So we take $f: W \rightarrow \mathbb{R}$ to be the function defined by setting $f(M)$ to be the value of $|\mathcal{U}(\mathcal{S})|$ on $M$ (for all $M \in W$ ). We have to show that for any $M, M^{\prime} \in W$ we have $\left|f(M)-f\left(M^{\prime}\right)\right| \leq d\left(M, M^{\prime}\right)$. To do so, we will construct a sequence $M_{0}, M_{1}, \ldots, M_{q}$ of matchings in $W$ such that $M_{0}:=M, M_{q}:=M^{\prime}$ and such that $M_{j}$ and $M^{\prime}$ agree on the first $j$ vertices in $\operatorname{Eff}\left(Q_{i}\right)$ (i.e. $M_{j}$ and $M^{\prime}$ have the same prefix). Suppose that we have constructed $M_{j}$ for some $j<q$ and that we now wish to construct $M_{j+1}$. Let $v$ be the first vertex in $\operatorname{Eff}\left(Q_{i}\right)$ on which $M_{j}$ and $M^{\prime}$ differ. Let $b$ be its neighbour in $M^{\prime}$ and let $v^{\prime}$ be the neighbour of $b$ in $M_{j}$. Define $M_{j+1}$ to be the matching obtained from $M_{j}$ by swapping the neighbours of $v$ and $v^{\prime}$ in $M_{j}$. So $M_{j+1}$ now agrees with $M^{\prime}$ on $v$ and all (the at least $j$ ) vertices preceding $v$ in $\operatorname{Eff}\left(Q_{i}\right)$. Note that $\left|f\left(M_{j}\right)-f\left(M_{j+1}\right)\right| \leq 4 \ell_{i}$ since swapping two edges can change $|\mathcal{U}(\mathcal{S})|$ by at most $4 \ell_{i}$. Indeed, to see the latter, note that for each one of these two edges there are $\ell_{i}-1$ other edges starting from the same path in $\mathcal{P}_{i}$, and therefore each of these two edges contributes to the degree of at most $\ell_{i}$ pairs in $\mathcal{U}_{i-1}^{\prime}$. If we swap these edges, this might change the degree of at most $4 \ell_{i}$ pairs. So $|\mathcal{U}(\mathcal{S})|$ can be increased or decreased by at most $4 \ell_{i}$. Also observe that $q \leq\left|M \triangle M^{\prime}\right| / 2$ since initially the number of vertices in $\operatorname{Eff}\left(Q_{i}\right)$ on which $M$ and $M^{\prime}$ differ equals $\left|M \triangle M^{\prime}\right| / 2$ and in each step this number decreases by at least 1 . Therefore,

$$
\left|f(M)-f\left(M^{\prime}\right)\right| \leq \sum_{j=0}^{q-1}\left|f\left(M_{j}\right)-f\left(M_{j+1}\right)\right| \leq 4 q \ell_{i} \leq 2 \ell_{i}\left|M \triangle M^{\prime}\right|=d\left(M, M^{\prime}\right) .
$$

Now, we are ready to apply (5): if $|\mathcal{U}(\mathcal{S})|>U_{i-1} / 27$, then by (30) we have $|\mathcal{U}(\mathcal{S})|$ $\mathbb{E}(|\mathcal{U}(\mathcal{S})|)>U_{i-1} /(2 \cdot 27)$ and (5) yields

$$
\begin{aligned}
& \mathbb{P}_{i}\left(|\mathcal{U}(\mathcal{S})|>\frac{U_{i-1}}{27}\right) \leq 2 \exp \left(-2 \frac{\left(U_{i-1} /(2 \cdot 27)\right)^{2}}{\left|Q_{i}\right|_{\text {eff }} 8^{2} \ell_{i}^{2}}\right) \\
& \text { 99, 10 , 14) } 2 \exp \left(-\frac{1}{2^{7} \cdot 27^{2}} \frac{\varepsilon^{8} n^{2}}{\left(27^{i-1}\right)^{2}} \frac{3^{i}}{(1+o(1)) \sqrt{2} \varepsilon n} \frac{1}{100^{2} 9^{i-1}}\right) \\
& \leq \quad 2 \exp \left(-\frac{2 \varepsilon^{8} n}{\left(3^{i-1}\right)^{7}}\right)
\end{aligned}
$$

Now we prove (32). In this case we can apply (51) with metric $d\left(M, M^{\prime}\right):=2 \Delta_{i-1}\left|M \triangle M^{\prime}\right|$ and $c:=8 \Delta_{i-1}$. Indeed, for each candidate branch set $B$ and each $P \in \mathcal{P}_{i}$ the removal/addition of a $B-P$ edge can only affect the degrees of those pairs in $\mathcal{U}_{i-1}^{\prime}$ which contain $B$. But there are at most $\Delta_{i-1}$ such pairs. Thus

$$
\begin{aligned}
\mathbb{P}_{i}\left(|\mathcal{U}(\mathcal{S})|>\frac{U_{i-1}}{27}\right) & \leq 2 \exp \left(-2 \frac{\left(U_{i-1} /(2 \cdot 27)\right)^{2}}{\left|Q_{i}\right|_{\mathrm{eff}} 8^{2} \Delta_{i-1}^{2}}\right) \\
& \leq 2 \exp \left(-\frac{U_{i-1}^{2}}{2^{7} \cdot 27^{2}} \frac{3^{i}}{k t} \frac{\varepsilon^{1 / 4} k^{2}}{(3 / 2)^{2(i-1)} U_{i-1}^{2}}\right) \\
& \stackrel{17}{\leq} 2 \exp \left(-\frac{1}{2^{7} \cdot 27^{2}} \frac{3 \varepsilon^{1 / 4}(1+o(1)) \sqrt{2} \varepsilon^{2}}{1 / \varepsilon}(4 / 3)^{i-1}\right) . \\
& \leq 2 \exp \left(-\varepsilon^{4}(4 / 3)^{i-1}\right) \leq 2 \exp \left(-\varepsilon^{4} 3^{(i-1) / 4}\right),
\end{aligned}
$$

as required. 
Define $\beta$ by

$$
\beta:=\left(\frac{\varepsilon^{8}}{2\left(3^{i-1}\right)^{7}}\right)^{2} .
$$

Lemma 11. For each $i$ with $3^{i-1} \leq n^{2 / 33}$ let $Y_{i}$ denote the number of all those subsets $\mathcal{S}$ of $\mathcal{P}_{i}$ with $|\mathcal{S}|=(1-\beta)\left|\mathcal{P}_{i}\right|$ for which $|\mathcal{U}(\mathcal{S})|>U_{i-1} / 27$. Then $\mathbb{P}_{i}\left(Y_{i}>0\right) \leq n^{-1 / 34}$.

Proof. Note that (12) and the restriction on $i$ together imply that $\beta\left|\mathcal{P}_{i}\right|=\Omega\left(n / 3^{16 i}\right)=$ $\Omega\left(n^{1 / 33}\right)$ and so we may treat it as an integer. (31) implies that

$$
\mathbb{E}_{i}\left(Y_{i}\right) \leq\left(\begin{array}{c}
\left|\mathcal{P}_{i}\right| \\
(1-\beta)\left|\mathcal{P}_{i}\right|
\end{array}\right) 2 \exp \left(-\frac{2 \varepsilon^{8} n}{\left(3^{i-1}\right)^{7}}\right)
$$

Note that $\left|\mathcal{P}_{i}\right| \leq n$. So

$$
\left(\begin{array}{c}
\left|\mathcal{P}_{i}\right| \\
(1-\beta)\left|\mathcal{P}_{i}\right|
\end{array}\right)=\left(\begin{array}{c}
\left|\mathcal{P}_{i}\right| \\
\beta\left|\mathcal{P}_{i}\right|
\end{array}\right) \leq\left(\frac{e}{\beta}\right)^{\beta\left|\mathcal{P}_{i}\right|} \leq \beta^{-2 \beta\left|\mathcal{P}_{i}\right|} \leq \beta^{-2 \beta n} .
$$

Now note that if $a>0$ is sufficiently small then $a \ln \left(a^{-1}\right) \leq a^{1 / 2}$. Thus

$$
\left(\begin{array}{c}
\left|\mathcal{P}_{i}\right| \\
(1-\beta)\left|\mathcal{P}_{i}\right|
\end{array}\right) \leq e^{2 \beta^{1 / 2} n \stackrel{34}{=}} \exp \left(\frac{\varepsilon^{8} n}{\left(3^{i-1}\right)^{7}}\right) \text {. }
$$

So if $3^{i-1} \leq n^{2 / 33}$ then

$$
\mathbb{P}_{i}\left(Y_{i}>0\right) \leq \mathbb{E}_{i}\left(Y_{i}\right) \stackrel{\sqrt[35]{\leq}}{\leq} 2 \exp \left(-\frac{\varepsilon^{8} n}{\left(3^{i-1}\right)^{7}}\right)=\exp \left(-\Omega\left(n^{19 / 33}\right)\right) \leq n^{-1 / 34},
$$

as required.

6.4. An upper bound on the degrees of the paths in $\mathcal{G}_{i}$. Let $d:=10^{6}$. We now estimate the probability that a given path $P \in \mathcal{P}_{i}$ joins at least $d$ unjoined pairs in $\mathcal{U}_{i-1}^{\prime}$.

Lemma 12. If $d=10^{6}, i \leq i_{0}$ and $i$ satisfies

$$
3^{i-1} \geq 1 / \varepsilon,
$$

then for every fixed $P \in \mathcal{P}_{i}$ we have $\mathbb{P}_{i}\left(d_{\mathcal{G}_{i}}(P) \geq d\right) \leq \beta / 3^{i}$.

Proof. Suppose that $C \subseteq \mathcal{U}_{i-1}^{\prime}$ is a set of size $d$ which lies in the neighbourhood of $P$ in the auxiliary graph $\mathcal{G}_{i}$. Let $\mathcal{B}(C)$ denote the set of candidate branch sets involved in the pairs from $C$. Note that

$$
\sqrt{2 d} \leq|\mathcal{B}(C)| \leq 2 d
$$

Moreover, $P \sim B$ for each candidate branch set $B \in \mathcal{B}(C)$. (Recall that this means that there is an effective vertex on $P$ that is matched to some vertex in $\operatorname{Eff}_{i}(B)$, where $\operatorname{Eff}_{i}(B)$ was the set of all those effective vertices in $B$ that are still available after the $(i-1)$ th stage.) Now let $\mathbf{B}$ be the collection of all the sets $\mathcal{B}$ of candidate branch sets such that for each $B \in \mathcal{B}$ there is a $B^{\prime} \in \mathcal{B}$ with $\left(B, B^{\prime}\right) \in \mathcal{U}_{i-1}^{\prime}$ and such that $b:=|\mathcal{B}|$ satisfies

$$
\sqrt{2 d} \leq b \leq 2 d
$$

Thus $\mathcal{B}(C) \in \mathbf{B}$ for any $C$ as above and hence

$$
\mathbb{P}_{i}\left(d_{\mathcal{G}_{i}}(P) \geq d\right) \leq \sum_{\mathcal{B} \in \mathbf{B}} \mathbb{P}_{i}(P \sim B \forall B \in \mathcal{B}) .
$$


To bound the latter probability, consider any $\mathcal{B} \in \mathbf{B}$, let $b:=|\mathcal{B}|$ and $s:=\sum_{B \in \mathcal{B}} \operatorname{eff}_{i}(B) \leq b t$. Recall that $x_{i-1}$ was the total number of all those effective vertices in the branch sets that are matched after the first $i-1$ stages. So

$$
\begin{aligned}
\mathbb{P}_{i}(P \sim B \forall B \in \mathcal{B}) & \leq\left(\begin{array}{c}
\ell_{i} \\
b
\end{array}\right) \frac{(s)_{b}}{\left(k t-x_{i-1}\right)_{b}} \leq\left(\frac{e \ell_{i}}{b}\right)^{b}\left(\frac{s}{k t-x_{i-1}}\right)^{b} \\
& \leq\left(\frac{e \ell_{i}}{b} \frac{b t}{k t-x_{i-1}}\right)^{b} \stackrel{18}{\leq}\left(\frac{e t \ell_{i}}{k t / 2}\right)^{b}=\left(\frac{2 e \ell_{i}}{k}\right)^{b} .
\end{aligned}
$$

In the second inequality, we used that $\frac{a-j}{b-j}<\frac{a}{b}$, for $0<j<a<b$. To bound $|\mathbf{B}|$, consider an auxiliary graph $\mathcal{A}_{i-1}$ whose vertex set is the set of candidate branch sets and whose edges correspond to the pairs in $\mathcal{U}_{i-1}^{\prime}$. Since $\mathcal{A}_{i-1}$ involves only edges/pairs from $\mathcal{U}_{i-1}^{\prime}$ its maximum degree is at most $\Delta_{i-1}$. Consider any $b$ as in (37). Note that each $\mathcal{B} \in \mathbf{B}$ with $|\mathcal{B}|=b$ corresponds to a subgraph $F$ of $\mathcal{A}_{i-1}$ which has order $b$ and in which no vertex is isolated. We claim that for all $q \leq b / 2$, the number of such subgraphs $F$ having precisely $q$ components is at most $U_{i-1}^{q}\left(b \Delta_{i-1}\right)^{b-2 q}$. To see this, note that each component of $F$ has to contain at least one edge (this is also the reason why it makes sense only to consider $q \leq b / 2$ ). So each subgraph $F$ as above can be obtained as follows. First choose $q$ (independent) edges of $\mathcal{A}_{i-1}$. The number of choices for this is at most $U_{i-1}^{q}$. Now successively add the remaining $b-2 q$ vertices to the existing subgraph without creating new components. At each step there are at most $b$ vertices $y$ to which a new vertex $z$ can be attached and once we have chosen $y$, there are at most $\Delta\left(\mathcal{A}_{i-1}\right) \leq \Delta_{i-1}$ choices for $z$, which proves the claim.

Let $\mathbf{B}_{b, q}$ be the set of all those $\mathcal{B} \in \mathbf{B}$ that have size $b$ and induce $q$ components in $\mathcal{A}_{i-1}$. Then

$$
\begin{aligned}
& \sum_{\mathcal{B} \in \mathbf{B}_{b, q}} \mathbb{P}_{i}(P \sim B \forall B \in \mathcal{B}) \stackrel{\sqrt{37}}{\leq} U_{i-1}^{q}\left(2 d \Delta_{i-1}\right)^{b-2 q}\left(\frac{2 e \ell_{i}}{k}\right)^{b} \\
& \underset{\leq}{[10], \underset{14}{\leq},[15]}\left(\frac{U_{0}}{27^{i-1}} \frac{1}{4 d^{2}} \frac{\varepsilon^{1 / 4} k^{2} 81^{i-1} 4^{i-1}}{U_{0}^{2}}\right)^{q}\left(2 d \frac{U_{0}}{\varepsilon^{1 / 8} k 9^{i-1} 2^{i-1}} \frac{2 e \cdot 100 \cdot 3^{i-1}}{k}\right)^{b} \\
& \stackrel{[13}{\leq} \quad\left(12^{i-1}\right)^{q}\left(\frac{2000 d}{\varepsilon^{1 / 8} 6^{i-1}}\right)^{b} \leq\left(\frac{4 \cdot 10^{6} d^{2}}{\varepsilon^{1 / 4} 3^{i-1}}\right)^{b / 2} \leq\left(\frac{1}{\varepsilon^{1 / 3} 3^{i-1}}\right)^{b / 2} \stackrel{\sqrt[36]{\leq}}{\leq}\left(\frac{\varepsilon^{1 / 6}}{3^{(i-1) / 2}}\right)^{b / 2} \text {. }
\end{aligned}
$$

Since $b \geq \sqrt{2 d} \geq 4 \cdot 48$ by (37) this implies

$$
\mathbb{P}_{i}\left(d_{\mathcal{G}_{i}}(P) \geq d\right) \stackrel{\sqrt{38} \leq}{\leq} \sum_{\sqrt{2 d} \leq b \leq 2 d} \sum_{q=1}^{b / 2}\left(\frac{\varepsilon^{1 / 6}}{3^{(i-1) / 2}}\right)^{b / 2} \leq 2 d^{2}\left(\frac{\varepsilon^{1 / 3}}{3^{i-1}}\right)^{\sqrt{2 d} / 4} \leq 2 d^{2}\left(\frac{\varepsilon^{1 / 3}}{3^{i-1}}\right)^{48} \leq \frac{\beta}{3^{i}}
$$

as required.

Given $d=10^{6}$ and $i$ satisfying (36), let $\mathcal{S}_{i}$ denote the set of paths in $\mathcal{P}_{i}$ which have degree less than $d$ in $\mathcal{G}_{i}$. We will now use Lemma 12 to show that with high probability $\mathcal{S}_{i}$ is large.

Lemma 13. $\mathbb{P}_{i}\left(\left|\mathcal{S}_{i}\right| \geq(1-\beta)\left|\mathcal{P}_{i}\right|\right) \geq 1-n^{-1 / 34}$ for every $i \leq i_{0}$ which satisfies (36).

Proof. Let $\overline{\mathcal{S}}_{i}:=\mathcal{P}_{i} \backslash \mathcal{S}_{i}$. Note that Lemma 12 implies

$$
\mathbb{E}_{i}\left(\overline{\mathcal{S}}_{i}\right)=\mathbb{P}_{i}\left(d_{\mathcal{G}_{i}}(P) \geq d\right)\left|\mathcal{P}_{i}\right| \leq \frac{\beta}{3^{i}}\left|\mathcal{P}_{i}\right|
$$


If $\left(\log _{3} n\right) / 34 \leq i \leq i_{0}$ then together with Markov's inequality this yields

$$
\mathbb{P}_{i}\left(\left|\overline{\mathcal{S}}_{i}\right|>\beta\left|\mathcal{P}_{i}\right|\right) \leq \frac{1}{3^{i}} \leq \frac{1}{n^{1 / 34}}
$$

and thus Lemma 13 holds for all such $i$.

If $i \leq\left(\log _{3} n\right) / 34$ we will use (5). As in the proof of Lemma 10, the underlying metric space will be the set of all those matchings which match the set $\operatorname{Eff}\left(Q_{i}\right)$ of effective vertices on $Q_{i}$ into the set $\bigcup_{j=1}^{k} \operatorname{Eff}_{i}\left(B_{j}\right)$ of all those effective vertices in the candidate branch sets that are still unmatched after the first $i-1$ stages. The series of partitions and the bijections $\phi$ are also as defined there. However, the metric imposed on $W$ now changes: for any $M, M^{\prime} \in W$ we set $d\left(M, M^{\prime}\right)=\left|M \triangle M^{\prime}\right|$. In particular this means that we can take $c:=4$. $f: W \rightarrow \mathbb{R}$ will be the function defined by taking $f(M)$ to be the value of $\left|\overline{\mathcal{S}}_{i}\right|$ on $M$ (for all $M \in W$ ). Note that the analogue of (33) is satisfied, since if we switch the endpoints of two edges of a matching (as it is the case when we obtain $M_{j+1}$ from $M_{j}$ as in the proof of Lemma 10) $\left|\overline{\mathcal{S}}_{i}\right|$ changes by at most 2 as switching two edges only affects the degree of the (at most) two paths involved. Thus

$$
\left|f(M)-f\left(M^{\prime}\right)\right| \leq \sum_{j=0}^{q-1}\left|f\left(M_{j}\right)-f\left(M_{j+1}\right)\right| \leq 2 q \leq\left|M \triangle M^{\prime}\right| \leq d\left(M, M^{\prime}\right) .
$$

Hence applying (5) with $a:=\beta\left|\mathcal{P}_{i}\right| / 2$ we obtain

$$
\mathbb{P}_{i}\left(\left|\overline{\mathcal{S}}_{i}\right| \geq \beta\left|\mathcal{P}_{i}\right|\right) \leq 2 \exp \left(-2 \frac{\beta^{2}\left|\mathcal{P}_{i}\right|^{2}}{4 \cdot 16\left|Q_{i}\right|_{\text {eff }}}\right)
$$

So to complete the proof, it suffices to show that $\beta^{2}\left|\mathcal{P}_{i}\right|^{2} /\left|Q_{i}\right|_{\mathrm{eff}}=\Omega\left(n^{3 / 34}\right)$, as this gives an error bound of $\exp \left(-\Omega\left(n^{3 / 34}\right)\right) \leq 1 / n^{34}$. To prove the former, note that by (9), (12) and (34) we obtain

$\frac{\beta^{2}\left|\mathcal{P}_{i}\right|^{2}}{\left|Q_{i}\right|_{\mathrm{eff}}}=\Theta\left(\frac{1}{\left(3^{i-1}\right)^{28}}\left(\frac{n}{9^{i-1}}\right)^{2} \frac{3^{i}}{n}\right)=\Theta\left(\frac{n}{3^{31(i-1)}}\right)=\Omega\left(\frac{n}{3^{31\left(\log _{3} n\right) / 34}}\right)=\Omega\left(n^{3 / 34}\right)$,

as required.

6.5. Finding a large matching of $\mathcal{G}_{i}$. The next lemma shows that with high probability we can join the required number of pairs from $\mathcal{U}_{i-1}^{\prime}$ during the $i$ th stage.

Lemma 14. For each $i \leq i_{0}$ we have $\mathbb{P}_{i}\left(\left|\mathcal{U}_{i}\right|>U_{i-1} / 27\right) \leq 2 n^{-1 / 34}$.

Proof. Recall that $\mathcal{U}_{i-1}^{\prime}$ (defined after (16) ) was obtained from $\mathcal{U}_{i-1}$ by discarding all those pairs containing a candidate branch set from $\mathcal{B}_{i}$. By definition of $\mathcal{U}_{i}$ we may assume that $\left|\mathcal{U}_{i-1}^{\prime}\right| \geq U_{i-1} / 27$ and it suffices to show that in $\mathcal{G}_{i}$ we can find a matching which covers all but at most $U_{i-1} / 27$ vertices/pairs in $\mathcal{U}_{i-1}^{\prime}$.

Case 1: $3^{i-1}<1 / \varepsilon$.

In this case, we apply (31) with $\mathcal{S}:=\mathcal{P}_{i}$ (i.e. $\alpha=1$ ) to obtain that with probability at least $1-2 \exp \left(-2 \varepsilon^{8} n /\left(3^{i-1}\right)^{7}\right) \geq 1-2 n^{-1 / 34}$ we have the following: there is a set $\mathcal{W} \subseteq \mathcal{U}_{i-1}^{\prime}$ with $|\mathcal{W}|=\left|\mathcal{U}_{i-1}^{\prime}\right|-U_{i-1} / 27$ so that every pair in $\mathcal{W}$ has degree at least $1 /\left(2 \varepsilon^{3}\right)$ in $\mathcal{G}_{i}$. On the other hand, clearly every path in $\mathcal{P}_{i}$ has degree at most $\ell_{i}^{2}=10^{4} 9^{i-1}<10^{4} / \varepsilon^{2}$ in $\mathcal{G}_{i}$. This implies that the subgraph $\mathcal{G}_{i}^{\prime}$ of $\mathcal{G}_{i}$ induced by $\mathcal{W}$ and $\mathcal{P}_{i}$ has a matching covering all of $\mathcal{W}$. To see this, consider any $\mathcal{W}^{\prime} \subseteq \mathcal{W}$ and let $N\left(\mathcal{W}^{\prime}\right) \subseteq \mathcal{P}_{i}$ denote its neighbourhood in $\mathcal{G}_{i}^{\prime}$. Then by counting edges between $\mathcal{W}^{\prime}$ and $N\left(\mathcal{W}^{\prime}\right)$ we obtain that $\left|\mathcal{W}^{\prime}\right| /\left(2 \varepsilon^{3}\right) \leq\left(10^{4} / \varepsilon^{2}\right)\left|N\left(\mathcal{W}^{\prime}\right)\right|$. 
This in turn implies that $\left|N\left(\mathcal{W}^{\prime}\right)\right| \geq\left|\mathcal{W}^{\prime}\right|$ and so Hall's condition is satisfied. But this means that we can take $\mathcal{U}_{i}:=\mathcal{U}_{i-1}^{\prime} \backslash \mathcal{W}$. Note that $U_{i}=\left|\mathcal{U}_{i-1}^{\prime}\right|-|\mathcal{W}|=U_{i-1} / 27$, as required.

Case 2: $1 / \varepsilon \leq 3^{i-1} \leq n^{2 / 33}$.

In this case we first apply Lemma 13 to see that with probability at least $1-n^{-1 / 34}$ we have $\left|\mathcal{S}_{i}\right| \geq(1-\beta)\left|\mathcal{P}_{i}\right|$. By taking a subset we may assume that $\left|\mathcal{S}_{i}\right|=(1-\beta)\left|\mathcal{P}_{i}\right|$. On the other hand, Lemma[11implies that with probability at least $1-n^{-1 / 34}$ any set $\mathcal{S}$ of this size satisfies $|\mathcal{U}(\mathcal{S})| \leq U_{i-1} / 27$. So with probability at least $1-2 n^{-1 / 34}$ we have $\left|\mathcal{U}\left(\mathcal{S}_{i}\right)\right| \leq U_{i-1} / 27$. But if this is the case then there is a set $\mathcal{W} \subseteq \mathcal{U}_{i-1}^{\prime}$ with $|\mathcal{W}|=\left|\mathcal{U}_{i-1}^{\prime}\right|-U_{i-1} / 27$ so that every pair in $\mathcal{W}$ has degree at least $1 /\left(2 \varepsilon^{3}\right)$ in the subgraph $\mathcal{G}_{i}^{\prime \prime}$ of $\mathcal{G}_{i}$ induced by $\mathcal{W}$ and $\mathcal{S}_{i}$. On the other hand, the definition of $\mathcal{S}_{i}$ implies that in $\mathcal{G}_{i}^{\prime \prime}$, the degree of every vertex in $\mathcal{S}_{i}$ is at most $d=10^{6}$. As in the previous case, this implies that $\mathcal{G}_{i}^{\prime \prime}$ has a matching covering all of $\mathcal{W}$. Indeed, to verify Hall's condition consider any $\mathcal{W}^{\prime} \subseteq \mathcal{W}$ and let $N\left(\mathcal{W}^{\prime}\right) \subseteq \mathcal{S}_{i}$ denote its neighbourhood in $\mathcal{G}_{i}^{\prime \prime}$. Then $\left|\mathcal{W}^{\prime}\right| /\left(2 \varepsilon^{3}\right) \leq 10^{6}\left|N\left(\mathcal{W}^{\prime}\right)\right|$. As before, we can take $\mathcal{U}_{i}:=\mathcal{U}_{i-1}^{\prime} \backslash \mathcal{W}$.

Case 3: $3^{i-1} \geq n^{2 / 33}$.

In this case, we apply (32) to $\mathcal{S}:=\mathcal{P}_{i}$ in order to obtain that with probability at least

$$
1-2 \exp \left(-\varepsilon^{4} 3^{(i-1) / 4}\right) \geq 1-2 \exp \left(-\varepsilon^{4} n^{1 / 66}\right) \geq 1-n^{-1 / 34}
$$

we have the following: there is a set $\mathcal{W} \subseteq \mathcal{U}_{i-1}^{\prime}$ with $|\mathcal{W}|=\left|\mathcal{U}_{i-1}^{\prime}\right|-U_{i-1} / 27$ so that every pair in $\mathcal{W}$ has degree at least $1 /\left(2 \varepsilon^{3}\right)$ in $\mathcal{G}_{i}$. On the other hand, Lemma 12 implies that the probability that $\mathcal{P}_{i}$ does not contain a path of degree at least $d=10^{6}$ in $\mathcal{G}_{i}$ is at least

$$
1-\frac{\beta\left|\mathcal{P}_{i}\right|}{3^{i}} \stackrel{12,(34)}{\geq} 1-O\left(n / 3^{17 i}\right)=1-O\left(n^{-1 / 33}\right) \geq 1-n^{-1 / 34} .
$$

So we may assume that both events occur and we get a matching covering all of $\mathcal{W}$ in the subgraph $\mathcal{G}_{i}^{\prime}$ of $\mathcal{G}_{i}$ induced by $\mathcal{W}$ and $\mathcal{P}_{i}$ as before. So we again obtain a set $\mathcal{U}_{i}$ of the desired size, with the required error bounds.

To complete the proof of Theorem 6 it remains to combine all the error probabilities for all the $i_{0}=\left(\log _{3} n\right) / 6$ stages. Recall that when analyzing the $i$ th stage we conditioned on $\mathcal{M}_{i-1}^{*}$ (defined after (19)). However, all our probability bounds hold regardless of what the actual value of $M_{i-1}^{*}$ is (as long as $M_{i-1}^{*}$ is good). So suppose that $\left|\mathcal{U}_{i-1}\right|=U_{0} / 27^{i-1}$ for some $i \leq i_{0}$. If $\left|\mathcal{U}_{i}\right| \neq U_{0} / 27^{i}$ then either some candidate branch set violated (19) or we had the undesired event that $\left|\mathcal{U}_{i}\right|>U_{i-1} / 27$ in Lemma 14. Thus

$$
\mathbb{P}\left(\left|\mathcal{U}_{i}\right|=U_{0} / 27^{i}|| \mathcal{U}_{i-1} \mid=U_{0} / 27^{i-1}, \mathcal{X}, \mathcal{X}^{\prime}\right) \geq 1-\exp \left(-\Omega\left(\ln ^{2} n\right)\right)-2 n^{-1 / 34} \geq 1-3 n^{-1 / 34}
$$

and so

$$
\mathbb{P}\left(\left|\mathcal{U}_{i}\right|=U_{0} / 27^{i} \forall i \leq i_{0} \mid \mathcal{X}, \mathcal{X}^{\prime}\right) \geq 1-3 i_{0} n^{-1 / 34} \geq 1-n^{-1 / 35} .
$$

This bound holds regardless of what the choices of $X_{1}, X_{2}, X_{1}^{\prime}, X_{2}^{\prime}$ actually are (as long as $\left|X_{1}\right|=\left|X_{2}\right|$ is within the range determined in Lemma 7). The only other reason why $\left|\mathcal{U}_{i_{0}}\right| \neq U_{0} / 27^{i_{0}}$ is that we had an undesired event in Lemma 7 . This happens with probability $O\left(1 / \ln ^{2} n\right)$. Altogether this shows that with probability $1-n^{-1 / 35}-O\left(1 / \ln ^{2} n\right)=1-o(1)$ after the $i_{0}$ th stage we are left with

$$
U_{i_{0}}=\frac{U_{0}}{27^{i_{0}}} \stackrel{8,(13)}{=} \varepsilon^{4} n^{1 / 2}
$$


unjoined pairs. We now discard a candidate branch set in each of these pairs as well as all the candidate branch sets in $\mathcal{B}_{0} \cup \cdots \cup \mathcal{B}_{i_{0}-1}$. By (17) and (39) this gives a complete minor on $k-6 \varepsilon^{1 / 8} k-\varepsilon^{4} n^{1 / 2} \geq \varepsilon^{2} n^{1 / 2}$ vertices, as required.

\section{ACKnowledgements}

We are grateful to Tomasz Łuczak for helpful discussions on the phase transition of $G_{n, p}$.

\section{REFERENCES}

[1] M. Ajtai, J. Komlós and E. Szemerédi, Topological complete subgraphs in random graphs, Studia Sci. Math. Hungar. 14 (1979), 293-297.

[2] E.A. Bender and E.R. Canfield, The asymptotic number of labelled graphs with given degree sequences, J. Combin. Theory A 24 (1978), 296-307.

[3] B. Bollobás, Random Graphs, 2nd. edition, Cambridge University Press, 2001.

[4] B. Bollobás, A probabilistic proof of an asymptotic formula for the number of labelled regular graphs, Europ. J. Combin. 1 (1980), 311-316.

[5] B. Bollobás, The evolution of random graphs, Trans. Amer. Math. Soc 286 (1984), 257-274.

[6] B. Bollobás and P.A. Catlin, Topological cliques in random graphs, J. Combin. Theory B 30 (1981), $224-227$.

[7] B. Bollobás, P.A. Catlin and P. Erdős, Hadwiger's conjecture is true for almost every graph, Europ. J. Combin. 1 (1980), 195-199.

[8] P. Erdős and S. Fajtlowicz, On the conjecture of Hajós, Combinatorica 1 (1981), 141-143.

[9] N. Fountoulakis, D. Kühn and D. Osthus, The order of the largest complete minor in a random graph, to appear in Random Structures and Algorithms.

[10] S. Janson, Random regular graphs: asymptotic distributions and contiguity, Combinatorics, Probability E Computing 4 (1995), 369-405.

[11] S. Janson, T. Łuczak and A. Ruciński, Random Graphs, Wiley Interscience, 2000.

[12] J. Kleinberg and R. Rubinfeld, Short paths in expander graphs, Proc. 37th Symposium on Foundations of Computer Science (FOCS), IEEE Comput. Soc. Press (1996), 86-95.

[13] M. Krivelevich and B. Sudakov, Minors in expanding graphs, preprint 2006.

[14] T. Łuczak, Component behavior near the critical point of the random graph process, Random Structures and Algorithms 1 (1990), 287-310.

[15] T. Łuczak, Cycles in a random graph near the critical point, Random Structures and Algorithms 2 (1991), 421-440.

[16] T. Euczak, B. Pittel and J.C. Wierman, The structure of a random graph at the point of the phase transition, Trans. Amer. Math. Soc. 341 (1994), 721-748.

[17] K. Markström, Complete minors in cubic graphs with few short cycles and random cubic graphs, Ars Combinatoria 70 (2004), 289-295.

[18] C.J.H. McDiarmid, On the method of bounded differences, In Surveys in Combinatorics, 1989 (Norwich, 1989), London Math. Soc. Lecture Note Ser., 141, Cambridge Univ. Press, Cambridge, 1989, 148-188.

[19] S. Plotkin, S. Rao and W. Smith, Shallow excluded minors and improved graph decompositions, Proceedings 5th ACM-SIAM Symposium on Discrete Algorithms (SODA), ACM Press, New York (1994), $462-470$.

[20] N. Wormald, Models of random regular graphs, In Surveys in Combinatorics, 1999 (Canterbury, 1999), London Math. Soc. Lecture Note Ser., 267, Cambridge Univ. Press, Cambridge, 1999, 239-298.

Nikolaos Fountoulakis, Daniela Kühn \& Deryk Osthus

School of Mathematics

University of Birmingham

Edgbaston

Birmingham

B15 2TT

UK

E-mail addresses: \{nikolaos, kuehn, osthus\}@maths.bham.ac.uk 